\title{
Clutch size and malaria resistance
}

SIR - It was suggested more than a decade ago that the cost of egg production may be a major factor in the evolution of clutch size in birds ${ }^{1}$, and that although this cost is a fundamental assumption of theories on the evolution of life-history patterns, it had not yet been adequately tested ${ }^{1,2}$. Manipulation of the rate of egg-laying is a prerequisite for demonstrating this cost, but has been considered technically difficult ${ }^{1}$. Here we provide the first experimental evidence for a trade-off between egg production and parasite defence in the great tit (Parus major), a classic model species in the study of life-history evolution.

We removed the first two eggs laid in half the nests of our breeding population. Females in this experimental group therefore compensated by laying, on average, one more egg than females in a control group ( $a$ in the figure), but raised, on average, one offspring less ( $b$ in the figure). Fourteen days after their chicks had hatched, we assessed the prevalence of Plasmodium in the peripheral bloodstream of females.

Plasmodium is a malarial parasite that is common in the population of birds we are studying, and which potentially impairs survival and future reproduction ${ }^{3,4}$ of its host. If there is a trade-off between egg production and parasite defence, one

a, Mean number of eggs ( \pm s.e.) laid by females of a control group ( $n=41$ broods) and an experimental group in which the first two eggs were removed the day of laying. ( $n=35$ broods). Females of the test group produced a larger clutch than females of the control group $(t=1.99 ; 74$ d.f.; $P_{2 \text {-tailed }}=0.05$ ). There was no difference between the two groups in mean hatching date (mean difference $=0.5$ days; $t=0.30 ; 67$ d.f.; $P_{2 \text { tailed }}=0.76$ ). $b$, Mean number of nestlings ( \pm s.e.) 14 days post-hatching in the control group $(n=37)$ and in the test group $(n=33)$. Females of the test group raised significantly fewer chicks than did females of the control group $\left(t=3.06 ; 68\right.$ d.f.; $P_{2 \text {-tailed }}=0.003$ ). c. Prevalence of Plasmodium spp. ( $\pm 95 \%$ confidence limits of binomial distribution ${ }^{12}$ ) in male (open bars) and female (shaded bars) great tits in the test group $(n=30)$ and the control group $(n=35)$. Manipulation significantly affected the prevalence of Plasmodium spp. in females $\left(\chi^{2}=6.49\right.$; 1 d.f.; $P=0.011$ ), but not in males $\left(\chi^{2}=0.30 ; 1\right.$ d.f.; $\left.P=0.59\right)$. $P$ arents were caught with a door trap at the nestbox when nestlings were 14 days old. A drop of blood was taken from the brachial vein and a thin smear produced on a glass slide. Slides were air-dried, fixed with absolute methanol and stained with Giemsa stain. Each slide was screened with a light microscope under oil immersion for $10 \mathrm{~min}$, and Plasmodium spp. scored as present or absent. The experiments were performed in a stable breeding pop-

ulation of great tits around the campus of the University of Lausanne, in spring 1995. would predict a higher prevalence of parasites in the females of the experimental group, as they laid a larger clutch. Prevalence in males of the experimental group should not increase, because the number of offspring to be raised is no greater than in the control group. We found that an increase in the clutch size by one egg leads to an increase in the prevalence of Plasmodium from $20 \%$ in the control females to $50 \%$ in the experimental females ( $c$ in the figure), thus supporting the hypothesis of a trade-off between egg production and parasite defence. As expected, there was no difference in the prevalence of Plasmodium in males of the two groups ( $c$ in the figure).

Evolutionary theory predicts that the optimal clutch size is the result of a tradeoff between current and future reproduction $^{2,5,6}$. At the physiological level, this trade-off predicts that parents investing heavily in their current offspring will have fewer resources to allocate to parasite defence, thereby impairing their future reproduction ${ }^{7}$. For birds, the studies in which clutch or brood size were experimentally manipulated after egg-laying have shown that the clutch size giving rise to the most recruits is larger than the most common clutch, but has produced only equivocal evidence for a general trade-off
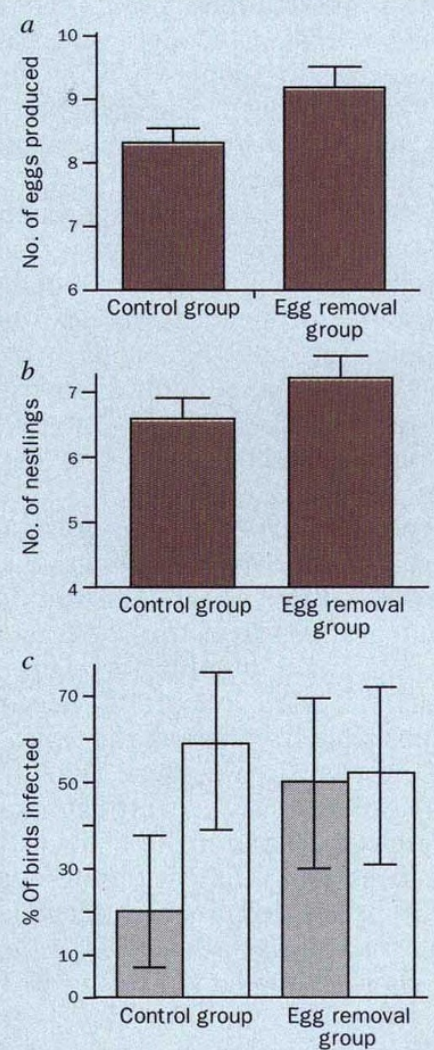

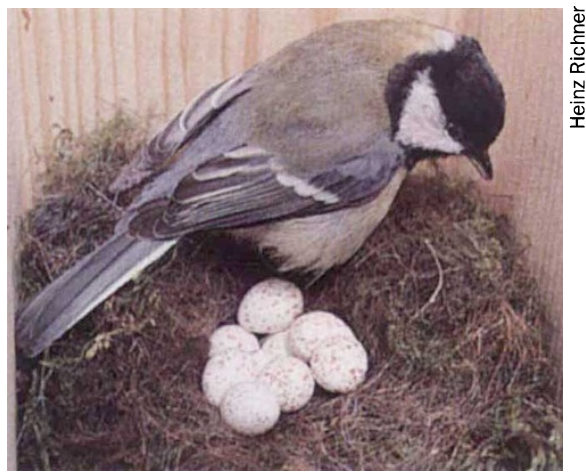

The cost of egg production in birds such as the great tit (Parus major) shown here may be an important factor in the evolution of clutch size.

in life-history between current and future reproduction $^{8-11}$.

It was stated recently that "all brood size manipulations ignore costs incurred earlier in the life history - in particular the cost of producing eggs, which might well affect the results of brood manipulation experiments" (p.158 of ref. 2). Our results support this contention, and provide evidence that the cost of egg production may be an important factor in the evolution of optimal clutch size in birds.

\section{Anne Oppliger*, Philippe Christe}

Zoology Department, IZEA,

University of Lausanne,

\section{CH-1015 Lausanne, Switzerland}

\section{Heinz Richner}

Zoology Department,

University of Bern,

CH-3032 Hinterkappelen, Switzerland

1. Partridge, L. \& Harvey, P. H. Nature 316, 20 (1985).

2. Stearns, S. The Evolution of Life Histories (Oxford Univ. Press, 1992).

3. Hayworth, A. M., van Riper, C. III \& Weathers, W. W. J. Parasit. 73, 850-853 (1987)

4. Richner, H., Christe, P. \& Oppliger, A. Proc. natn. Acad. Sci. U.S.A. 92, 1192-1194 (1995).

5. Williams, G. C. Am. Nat. 100, 687-690 (1966).

6. Charnov, E. L. \& Krebs, J. R. Ibis 116, 217-219 (1974)

7. Gustafsson, L. et al. Phil. Trans. R. Soc. B346, 323-331 (1994).

8. Gustafsson, L. \& Sutherland, W. J. Nature 335, 813-815 (1988)

9. Lindén, M. \& Møller, A. P. Trends Ecol. Evol. 4, 367-371 (1989).

10. Lessels, C. M. in Behavioural Ecology (eds Krebs, J. R. \& Davies, N. B.) 32-68 (Blackwell, Oxford, 1991).

1. Pettifor, R. J. Anim. Ecol. 62, 145-159 (1993).

12. Krebs, C. J. Ecological Methodology (Harper Collins, New York, 1989).

*The order of the authors was determined by tossing a coin. Correspondence should be addressed to H.R.

\section{Erratum}

IN the Scientific Correspondence "Roots in mixotrophic algae" published in the 30 May issue (Nature 381, 382; 1996), the authors' affiliations were transposed. They are shown correctly below.

\section{J. R. M. Chisholm ${ }^{*}$, C. Dauga ${ }^{\dagger}$ \\ E. Ageron ${ }^{\dagger}$, P. A. D. Grimont ${ }^{\dagger}$}

J. M. Jaubert ${ }^{\star}$

* Observatoire Océanologique Européen, Centre Scientifique de Monaco, MC 98000 Principality of Monaco †Unité des Entérobactéries, Unité 386 INSERM, Institut Pasteur, 75724 Paris Cedex 15, France 